\title{
The Real World or Web Engineering?
}

\author{
Lutz Heuser \\ Vice President Global Research \& Innovation \\ SAP AG
}

A couple of years ago practitioners and researchers began to realize that the traditional issues covered in software engineering do not fulfil all of the requirements raised by the proliferation and growth of web-based information and application systems.

"Fundamental differences [between hypermedia and other disciplines] however, make a pure transposition of techniques both difficult and inadequate. An important part of hypertext design concerns aesthetic and cognitive aspects that software engineering environments do not support,"

[Nanard and Nanard, 1995]

As a result, researchers currently make significant and ongoing contributions to a discipline that is called Web Engineering (WebE). Web Engineering aims to address and resolve the multifaceted problems of Web-based systems development. Even there are quite some different definitions for Web Engineering, the main focus of is usually on the establishment and use of sound scientific, engineering, and management principles and systematic approaches to the successful development, deployment and maintenance of high-quality Web-based systems and applications [San Murugesan, Yogesh Deshpande: Workshop Web Engineering ICSE2000].

Web Engineering is the application of systematic, disciplined and quantifiable approaches to the cost-effective development and evolution of high-quality applications in the World Wide Web.

[www.webengineering.org, March 2004]

\begin{abstract}
"Web Engineering is a discipline among disciplines, cutting across computer science, information systems, and software engineering, as well as benefiting from several non-IT specializations"

"While Web Engineering adopts and encompasses many software engineering principles, it incorporates many new approaches, methodologies, tools, techniques, and guidelines to meet the unique requirements of Web-based systems. Developing Web-based systems is significantly different from traditional software development and poses many additional challenges"
\end{abstract}

[IEEE Multimedia]

After taking a closer look at the Web Engineering principles and approaches that have already been developed, we recognise that most of these concepts assume that 
systems and applications are developed from scratch and that the target environment is the smaller/mid-size business environment.

Both of these assumptions do not apply to many of the established software companies and especially not to SAP. Founded in 1972, SAP is the recognized leader in providing collaborative business solutions for all types of industries and for every major market. SAP solutions are installed at more than 60,000 customer locations in 120 countries. Comprehensive solutions are currently available for 23 distinct industries and each solution is tailored to the specific standards, processes, and challenges of the respective industry. And they're developed, implemented, and supported by 28,700 professionals operating out of a global network of offices.

Thanks to SAP's unified technology platform, all solutions share common values, including:

- Seamless integration: Removes the barriers that stand between people, systems, and information

- Scalability: Accommodates virtually unlimited growth

- Adaptability: Allows easy customization of features and functions - and helps to cope with constant change

- Ease of implementation: Helps the customer to get up and running sooner

- Lower total cost of ownership: Helps minimize long-term costs

- Industry expertise: Supports the real-world processes employed every day

As SAP has always been committed to the development of technology-independent business solutions, the evolution from the mainframe-based R/2 system to R/3 for Client/Server and Distributed Open Systems was just as natural as the progression towards web-based systems.

Now SAP has announced that the future product portfolio will be based on an enterprise service architecture utilising web services on common business objects and business processes. This requires a "real world" definition of what a Web Service could be.

SAP's software engineering process is based on the Product Innovation Lifecycle (PIL), which describes how SAP invents, produces, and manages products throughout their entire lifetime from invention to transition and across multiple releases. The PIL is primarily driven by Portfolio \& Solution Management and involves virtually all of SAP's line of business functions (e.g. development, production, support).

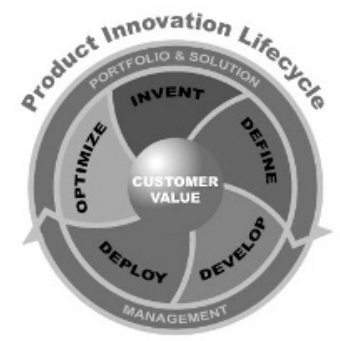

Fig. 1. SAP's Product Innovation Lifecycle 
The SAP Product Innovation Lifecycle (PIL) is based on a waterfall model that can be complemented by spiral/parallel approaches within PIL phases as needed.

The deliverable-oriented phases of the PIL transform ideas into software releases, which in turn are deployed to create successful implementations.

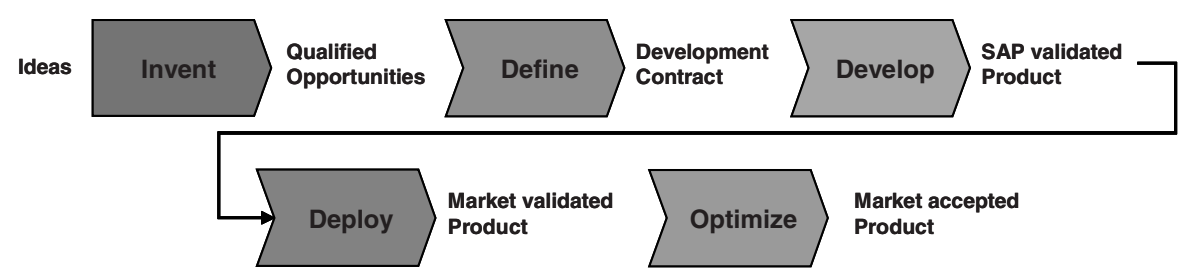

Fig. 2. Information flow in the PIL

Having said this, the following sections will sketch some of the key challenges in the discipline of Web Engineering where SAP would like to evaluate how the concepts of numerous ambitious and skilful researchers could be implemented in the real world. The examples also outline how the scientific Web Engineering phases could be mapped to SAP's PIL.

\section{Example: WebE: Requirement Engineering / PIL: Invent, Define}

Successfully defining the stakeholders' requirements is critical to the development of an application that delivers quality and meets expectations. Difficulties during the development process or creation of an unsuccessful application usually result from mistakes such as missing or not well-defined requirements as well as inclusion of superfluous requirements.

In the real world, application development is often not carried out for a single customer but for a market segment. Standard applications have to fulfil the needs for a superset of customers of a specific business area (e.g. Enterprise Resource Planning, Human Resources) or coming from a specific industry (e.g. Automotive, Retail).

Another real-world aspect that has to be addressed is the prioritisation of requirements according to the context of use with respect to the user as well as the application. Additionally, changes and advances in technology (e.g. user agents, standards, protocols) dynamically influence the set and prioritisation of requirements.

Starting with a wide array of concepts and tools for the selection of relevant requirements, the Requirement Engineering phase is arguably lacking in well-defined and systematic support for the each of its components, which include the formal description, validation, management, and risk assessment of requirements as well as the cost forecasting/estimation analysis.

Within SAP, it is agreed that any development to be initiated requires a so-called business case. It clearly defines why the development should be done in terms of customer demands or market opportunities. It is also necessary to justify required investments and to position the possible solution in relation to the competition. 


\section{Example: WebE: Analysis, Design / PIL: Define, Develop}

For the analysis and design aspects of Web Engineering, the initial steps in the PIL Development phase are the detailed planning and the detailed design. Keeping in mind that SAP products are used at 60,000 customer locations in more than 120 countries, the design of the applications has to pay close attention to unique cultural aspects, localisation, and configuration of the local IT infrastructures.

Web Engineering often arranges the analysis and design activities into orthogonal dimensions for content, interaction, navigation, presentation, processes, and communication. However, the reality is that this orthogonal categorization doesn't necessarily map well to existing systems and earlier development, as they are often stored in legacy systems, follow earlier process models, or have been set by the customer.

In addition, current scientific approaches often do not fully take changing technologies and even changing development and architectural paradigms (e.g. client/server towards enterprise service architectures) into consideration when establishing analysis and design concepts for broad use.

With respect to these aspects, the implementation of the Analysis and Design concepts developed in Web Engineering, have not been previously implemented very often because they do not take into account pre-existing legacy systems and development and sometimes neglect the reality that technology is constantly evolving.

\section{Example: WebE: Implementation / PIL: Develop}

In 1992, Derek Patridge stated, "Conventional software-system development is the process of designing a correct HOW for a well-defined WHAT." Whereas the "WHAT" has already been defined in the previous phases of the Web Engineering process, the "HOW" should now clarify details about the implementation.

As a result, emerging Web Engineering concepts often try to answer this by answering the question regarding "HOW the latest Web-technologies (e.g. SMIL, XForms, XSL-FO) could be applied." Whenever a new version of a particular Webtechnology appears, the answer it provides is usually a new and of course improved solution for the "HOW" compared to the previous version.

In the real world, Partridge's question about the "HOW" could meanwhile be rephrased as, "HOW all of these technologies should be combined and HOW to keep pace with the technological evolution?" Neither concepts that have been previously developed nor the tools that are currently being used allow developers and researchers to use the latest technology and to use them in conjunction with legacy technologies.

\section{Conclusion}

Beginning with the provoking question, "The real world or Web Engineering?" this brief overview has provided on one hand a short review of the roots of the discipline of Web Engineering and on the other hand introduced SAP's background and Product Innovation Lifecycle. Three examples also demonstrated where a mismatch between SAP's real world requirements and scientific Web Engineering results could be seen. 
The conclusion however is that Web Engineering is a scientific discipline with a solid theoretical basis that delivers valuable and important pieces in solving the puzzle of how to build large, complex web-based systems.

Research opportunities have been identified to answer questions such as, "How should these pieces be put together?" and "What is the best way to deal with the rapid evolution of technology and the resulting heterogeneity of these systems?" 